\title{
Strömgren four-color photometry of X-ray active late-type stars: Evidence for activity-induced deficiency in the $m_{1}$ index ${ }^{\star}$
}

\author{
F. Morale ${ }^{1}$, G. Micela ${ }^{1}$, F. Favata ${ }^{2}$ and S. Sciortino ${ }^{1}$ \\ 1 Istituto e Osservatorio Astronomico di Palermo, Palazzo dei Normanni, 90134 Palermo, Italy \\ 2 Astrophysics Division, European Space Agency - Postbus 299, 2200 AG Noordwijk, The Netherlands
}

Received October 30, 1995; accepted February 9, 1996

\begin{abstract}
We present the results of a $u v b y-\beta$ photometric study of a sample of active late-type stars ( $\mathrm{F}-\mathrm{K}$ ) selected from the Einstein Extended Medium Sensitivity Survey. Our work shows the presence in the sample of a star population with the photometric index $c_{1}$ typical of main sequence stars and an unexpected deficiency in $m_{1}$ index. Stars with more anomalous values of $m_{1}$ have also very high values of $f_{\mathrm{X}} / f_{\mathrm{V}}$ and X-ray surface fluxes, near the "saturation" limit observed in the most active stars and similar to the flux observed in solar active regions. We discuss these results in the light of similar results found in the Sun comparing $m_{1}$ indices in quiet and active regions and in other samples of active stars.
\end{abstract}

Key words: stars: activity — stars: late-type — stars: abundances — X-rays: stars

\section{Introduction}

X-ray flux limited surveys have been a very powerful medium for the study of the characteristics of known populations of coronal emitters, as well as for the discovery of new populations with unremarkable optical characteristics, which would therefore be difficult to detect otherwise. One of the most productive surveys has been the Extended Medium Sensitivity Survey (EMSS, Gioia et al. 1990), an X-ray flux limited survey based on data acquired with the Imaging Proportional Counter on board the Einstein observatory, which constitutes to date the largest published unbiased and essentially fully identified sample of X-ray sources.

By comparing the contents of the EMSS with expected source counts based on Galaxy models Favata et al. (1988) and Sciortino et al. (1995) have shown the existence of an excess population of yellow stars in the EMSS with respect to the source counts expected for normal main sequence stars. Favata et al. (1993, 1995), studying the optical counterparts, have shown that this excess is largely due to a young (i.e. with an age close to ZAMS) stellar population which appears to be under-represented in the solar neighborhood. A similar conclusion has been reached by Tagliaferri et al. (1994) studying the High Latitude X-ray EXOSAT survey. Also, Jeffries \& Bromage (1993)

Send offprint requests to: F. Morale

${ }^{\star}$ Based on observations collected at the ESO La Silla observatory and Hodgkin \& Pye (1994) have studied the excess of EUV sources they found by comparing EUV luminosity functions deduced from ROSAT WFC observations of nearby stellar sources with the $\log (N)-\log (S)$ derived from the EUV Bright Source Catalog (Pounds et al. 1993), reaching similar conclusions.

To further investigate the nature of the solar-type stellar populations in the EMSS a photometric campaign in the Strömgren-Crawford $u v b y-\beta$ system was performed, on a sample of EMSS sources, at the same time as the spectroscopic observations reported by Favata et al. (1993). The original primary aim of these observations was to determine effective temperatures and luminosity classes for the observed sources. In addition to fulfilling this aim, a detailed study of the $m_{1}$ color index in our sample has allowed us to explore the effect of activity on the "traditional" metallicity photometric indicator $m_{1}$, which had already been shown to deviate from the expected behavior in samples of very active stars (see for example Giménez et al. 1991).

Strömgren photometry has been a very powerful tool for the investigation of several important questions about stellar populations and Galactic stellar structure. Following the large body of observational work on early type stars (from B to early G), which has yielded important calibrations of fundamental astrophysical quantities (Crawford 1975, 1978, 1979; Nissen 1981; McNamara \& Powell 1985; Saxner \& Hammarbäck 1985; Olsen 1988; Nissen \& Schuster 1991), more recently a large effort 
has been directed toward enlarging the applicability of the calibrations toward later type stars (Ardeberg \& Lindgren 1981; Olsen 1984; Ardeberg \& Lindgren 1985; Reglero et al. 1990). We have fruitfully tapped into this large body of recent work for the analysis presented here.

The present paper is organized as follows: in Sect. 2 we describe the observed sample, in Sect. 3 we present the observations and the data reduction and analysis, in Sect. 4 we present our results, and in Sect. 5 we discuss our findings, in particular the meaning of the $m_{1}$ color in very active stars. Our main conclusions are summarized in Sect. 6.

\section{Program stars}

The sample discussed in the present paper is composed of 49 yellow stars selected from the coronal sources detected in the EMSS. They were selected in the color range $B-V$ comprised between 0.4 and 1.4 and are brighter than $\mathrm{V}$ magnitude $\approx 12$. Apart from the color and magnitude cuts, the only additional selection criterion imposed on the sample has been the visibility, i.e. the stars in the sample were visible from the ESO La Silla observatory, with the Danish $50 \mathrm{~cm}$ (Strömgren Automatic Telescope, SAT) during the observational campaign (August 1991). All the stars in the sample have measured X-ray fluxes from the EMSS, and a large fraction of them (32 out of 49) have had their lithium abundances and rotational velocities spectroscopically determined in the course of the same observational campaign (Favata et al. 1993). Additionally, several of the sample stars (22) have been studied by searching for companions through radial velocity measurements (Fleming 1989; Stocke 1991; Baker et al. 1994). Table 1 summarizes the main known characteristics of the program stars.

As expected given the nature of the selection bias imposed by X-ray flux limited survey, our sample is dominated by very active stars, on the high luminosity tail of the X-ray luminosity distribution.

\section{Observations and data reduction}

\subsection{Observations}

All the measurements presented here were obtained with the SAT telescope at the ESO La Silla observatory. The SAT is a $0.50 \mathrm{~m}$ Cassegrain telescope, devoted to uvby and $\beta$ photoelectric photometry, with computer programmed capabilities for the automatic execution of the observation program (Olsen 1994). The photometer is a six channel, microprocessor controlled instrument, and has dead time constants of $72,95,88$ and $73 \mathrm{~ns}$ for the $u, v, b, y$ channels, of $71 \mathrm{~ns}$ for the $\mathrm{H} \beta \mathrm{N}$ channel, and of $89 \mathrm{~ns}$ for the $\mathrm{H} \beta \mathrm{W}$ channel (Schwarz \& Melnick 1989).

The data discussed here were collected during seven nights in the first week of August 1991, using a 17 arcsec diaphragm for both the uvby and the $\beta$ channels. The integration time for program stars was chosen to obtain, for stars to $V=10$ (corresponding to the bulk of our sample) a $S / N$ ratio of 100 in the photometer $u$ and $\beta$ channels. The $S / N$ is usually higher in the other channels.

The rate of standard star (uvby and $\beta$ ) measurements was of 4 or 5 per hour, and some of those stars were observed in uvby up to an airmass 2 , to measure atmospheric extinction effects during the night. The sky background was observed after every star, both for the uvby and the $\beta$ measurements.

\section{2. uvby data reduction}

We have analyzed the data in two steps: first we have computed the instrumental catalog, determining the various instrumental and observational factors that contribute to the results, and then we transformed the data to the Crawford-Barnes (1970) standard values.

First of all, to obtain the extra-atmospheric magnitude and colors for standard stars, we have minimized a multi-night, multi-star merit function, with time drift terms and extinction coefficients, built according to the suggestions of Manfroid \& Heck (1983) and Sterken \& Manfroid (1992), fixing at least a mean term to roughly reduce our catalog to standard values. We then reduced this preliminary flexure-dependent instrumental catalog to the standard values listed in Table 8 of Olsen (1993) through Crawford \& Barnes (1970) expressions, modified to take into account the instrumental flexure errors. (the NOAOIRAF fitparams task was mostly used for the purpose). The flexure coefficients determined here, shown in Table 2, are different from the values of Olsen $(1993,1994)$, while the other transformation coefficients are very similar to the values of Olsen (1993).

After applying the flexure correction to the magnitude (y) and to the color data, we have used the corrected values to recompute the merit function and to finally derive our instrumental catalog for the SAT in August 1991, as shown in Table 3 .

A comparison between our catalog and Olsen's (1993) does not reveal systematic effects, with the exception of a small zero point shift. Therefore we are confident that the SAT instrument did not undergo substantial changes between 1987 (the date of Olsen's observations) and 1991. The root mean squares (RMS) residuals are 0.007, 0.004, $0.006,0.007,0.004$ for $y,(b-y), m_{1}, c_{1}$ and $\beta$, respectively.

In the transformation from instrumental to standard values a crucial step is the dependence on color that, for Strömgren photometry, is different for early and late type stars and among late type stars is furthermore different for main sequence and giants stars (Gronbech et al. 1976; Olsen 1983, 1984, 1993, 1994; Sterken \& Manfroid 1992).

Taking advantage of the good agreement between our and Olsen's (1993) instrumental catalogs and of the similarity between our transformation coefficients (determined 
Table 1. Optical characteristics of program stars

\begin{tabular}{|c|c|c|c|c|c|c|c|}
\hline$\#$ & EMSS Name & Other name & $V^{1}$ & Spec. type ${ }^{1}$ & $\log n(\mathrm{Li})^{2}$ & $\begin{array}{l}v \cdot \sin i^{3} \\
(\mathrm{~km} / \mathrm{s})\end{array}$ & Binary? \\
\hline 1 & MS0002.8+1602 & HD 42 & 8.6 & F5V & 2.50 & 11 & $\mathrm{~B}$ \\
\hline 2 & MS0003.3-4201 & HD 105 & 7.4 & G0V & 3.40 & 13 & \\
\hline 3 & MS0009.9+1417 & $\mathrm{BD}+1313$ & 8.5 & G5V & 1.35 & 25 & B \\
\hline 4 & MS0031.9-0646 & HD 3126 & 6.8 & F4V & 2.95 & 27 & \\
\hline 5 & MS0138.0-5627 & HD $10360 / 10361$ & 5.8 & $\mathrm{~K} 5 \mathrm{~V} / \mathrm{K} 0 \mathrm{~V}$ & -0.95 & 11 & B \\
\hline 6 & MS0206.2-1019 & & 8.9 & G5V & 0.95 & 19 & \\
\hline 7 & MS0234.2-0321 & HD 16287 & 8.1 & $\mathrm{~K} 2 \mathrm{~V}$ & -0.05 & 11 & $\mathrm{~B}$ ? \\
\hline 8 & MS0234.7-0210 & & 10.4 & G9V & 1.00 & 10 & \\
\hline 9 & MS0236.4-0148 & HD 16525 & 9.0 & F8V & 2.25 & & $\mathrm{~B} ?$ \\
\hline 10 & MS0244.8-0024 & BD-00431 & 9.6 & G9V & 1.10 & $\leq 8$ & B \\
\hline 11 & MS0300.1-1528 & HD 18955 & 8.4 & G8V & 0.50 & 20 & $\mathrm{~B}$ ? \\
\hline 12 & MS0318.5-1926 & & 10.8 & $\mathrm{~K} 7 \mathrm{~V}$ & 1.05 & 17 & \\
\hline 13 & MS0324.1-2012 & & 10.5 & G4V & & & \\
\hline 14 & MS0326.6-2008 & BD-20 646 & 10.1 & F4V & 2.90 & 15 & $\mathrm{~B} ?$ \\
\hline 15 & MS0327.2-2416 & HD 21703 & 9.1 & $\mathrm{~K} 7 \mathrm{~V}$ & & 14 & \\
\hline 16 & MS0333.1+0607 & & 10.3 & F6V & 3.10 & 33 & \\
\hline 17 & MS0337.6-0202 & HD 22853 & 8.0 & G9V & 1.20 & 18 & \\
\hline 18 & MS0348.2-1404 & & 10.7 & K0IV & 1.95 & $\leq 8$ & $\mathrm{~B} ?$ \\
\hline 19 & MS0356.9+1011 & HD 25102 & 6.4 & F5V & & & \\
\hline 20 & MS0420.3-3900 & & 12.0 & F6V & & & \\
\hline 21 & MS0438.5+0213 & & 10.6 & F9V & 0.65 & 48 & $\mathrm{~B} ?$ \\
\hline 22 & MS0452.2+0225 & & 10.6 & K0V & 1.40 & $\leq 8$ & $\mathrm{~B}$ ? \\
\hline 23 & MS0457.5+0312 & HD 25102 & 7.5 & $\mathrm{~K} 2 \mathrm{~V}$ & 2.05 & $\overline{3} 7$ & \\
\hline 24 & MS0515.4-0710 & & 10.8 & $\mathrm{~K} 2 \mathrm{e}$ & 2.70 & 11 & \\
\hline 25 & MS1247.0-0548 & HD 111487 & 9.7 & G5 & & & B \\
\hline 26 & MS1254.8+0142 & HD 112542 & 7.0 & F4V & 2.40 & 28 & \\
\hline 27 & MS1330.5-0811 & HD 117860 & 7.2 & G0V & 2.55 & $\leq 8$ & \\
\hline 28 & MS1335.9-2918 & HD 118646 & 5.8 & F5V & & & \\
\hline 29 & MS1428.2+0732 & & 11.0 & F7V & & & $\mathrm{B}$ ? \\
\hline 30 & MS1436.8-2628 & SAO 182743 & 9.7 & $\mathrm{~K} 4 \mathrm{~V}$ & -0.05 & 10 & $\mathrm{~B} ?$ \\
\hline 31 & MS1457.0+2108 & & 11.1 & F8V & & & \\
\hline 32 & MS1520.2+2548 & HD 136901 & 7.4 & K1III & & & B \\
\hline 33 & MS1528.5+0844 & HD 138290 & 6.5 & F4V & & & $\mathrm{B}$ ? \\
\hline 34 & MS1533.0+0919 & & 11.7 & $\mathrm{~K} 4 \mathrm{Ve}$ & & & \\
\hline 35 & MS1552.0-2338 & HD 142361 & 8.8 & G3IV & 4.05 & 53 & \\
\hline 36 & MS1558.4-2232 & & 11.4 & K3e & 3.10 & $\leq 8$ & \\
\hline 37 & MS1559.2-2232 & & 11.3 & K5e & & & $\mathrm{B} ?$ \\
\hline 38 & MS1753.5+1830 & GJ 698 & 9.2 & K4 & 0.75 & $\leq 8$ & $\mathrm{~B}$ ? \\
\hline 39 & MS1758.9+2339 & & 10.7 & $\mathrm{~K} 3 \mathrm{~V}$ & & & \\
\hline 40 & MS1907.0-6405 & & 11.8 & $\mathrm{~K} 4 \mathrm{~V}$ & 0.05 & 21 & \\
\hline 41 & MS2038.3-0046 & HD 197010 & 9.3 & F8V & & & $\mathrm{B}$ ? \\
\hline 42 & MS2119.7+1655 & & 11.7 & F6V & & & $\mathrm{B}$ ? \\
\hline 43 & MS2125.5-1503 & & 11.4 & F8V & & & $\mathrm{B}$ ? \\
\hline 44 & MS2148.2+1420 & & 11.4 & $\mathrm{~K} 3 \mathrm{IV} / \mathrm{V}$ & 1.60 & $\leq 8$ & $\mathrm{~B} ?$ \\
\hline 45 & MS2254.2+0219 & & 10.2 & $\mathrm{~K} 2 \mathrm{~V}$ & 1.15 & 43 & \\
\hline 46 & MS2302.4-4427 & HD 218033 & 9.7 & G8 & 1.25 & 16 & \\
\hline 47 & MS2315.1-3640 & & 11.3 & $\mathrm{~K} 2 \mathrm{~V} / \mathrm{K} 4 \mathrm{~V}$ & & 12 & \\
\hline 48 & MS2335.2+0305 & HD 222111 & 7.3 & F5V & 1.75 & 11 & \\
\hline 49 & MS2349.8-0112 & & 10.7 & KOIII & 3.75 & 64 & \\
\hline
\end{tabular}

${ }^{1}$ Data from Stocke et al. (1991); ${ }^{2}$ Data from Favata et al. (1993); ${ }^{3}$ Data from Favata et al. (1995).

in the course of the determination of flexure effects) and Olsen's (1993), we have decided to use the transformation of Olsen, which was obtained by using a larger number of standard stars than ours, after adding a zero point correction of few millimagnitudes to our instrumental catalogue, in order to match the values reported in Table 3 of Olsen (1993).

All the transformations determined by the reduction process described above were applied to the program stars together with the color transformation based on the deci- sion procedure described in Fig. 1 of Olsen (1993), which has made possible to perform a luminosity classification on the basis of the instrumental colours $(b-y)$ and $c_{1}$.

The catalogue of standard stars reduced to the Crawford-Barnes standard values is shown in Table 4. The standard values of program stars are listed in Table $5^{1}$,

${ }^{1}$ On June 15 1991, some weeks before our observations, the Pinatubo volcano $\left(\approx+15^{\circ}\right.$ latitude $)$, in the Philippines, underwent an explosive eruption launching a great mass of ashes and gases in atmosphere. Effects of volcano eruptions 
where the number of observations in the $y$ channel, $n_{y}$, and of observations for the colors, $n_{u v b}$, can be different because some non-photometric parts of nights, which are not usable for magnitude determinations, are however usable for color measurements when using a simultaneous data collecting photometer like the SAT (cf. Olsen 1983, 1993; Schuster \& Nissen 1988).

Table 2. Flexure coefficients for the gradient in declination $(\delta)$ and in hour angle $(h)$. Values and errors are the result of a minimization procedure. The correction to be applied to an observed index is $-K \delta$ and $-L h$

\begin{tabular}{|ccccc|}
\hline$K(y)$ & $K(b-y)$ & $K\left(m_{1}\right)$ & $K\left(c_{1}\right)$ & $K(\beta)$ \\
$\mathrm{mag} / \mathrm{rad}$ & $\mathrm{mag} / \mathrm{rad}$ & $\mathrm{mag} / \mathrm{rad}$ & $\mathrm{mag} / \mathrm{rad}$ & $\mathrm{mag} / \mathrm{rad}$ \\
\hline-0.0117 & +0.0030 & -0.0017 & +0.0075 & -0.0061 \\
\pm 0.0013 & \pm 0.0009 & \pm 0.0011 & \pm 0.0017 & \pm 0.0030 \\
\hline$L(y)$ & $L(b-y)$ & $L\left(m_{1}\right)$ & $L\left(c_{1}\right)$ & $L(\beta)$ \\
$\mathrm{mag} / \mathrm{hour}$ & $\mathrm{mag} / \mathrm{hour}$ & $\mathrm{mag} / \mathrm{hour}$ & $\mathrm{mag} / \mathrm{hour}$ & $\mathrm{mag} / \mathrm{hour}$ \\
\hline+0.00011 & -0.00006 & +0.00002 & +0.00024 & 0.00008 \\
\pm 0.00005 & \pm 0.00004 & \pm 0.00004 & \pm 0.00006 & \pm 0.00013 \\
\hline
\end{tabular}

\subsection{The $\beta$ data reduction}

To reduce $\beta$ data we followed the same procedure described above for the uvby data: we first minimized a merit function, similar to that adopted for uvby measurements but without the extinction term and we then built a preliminary instrumental catalog forcing the data to Crawford-Mander standard values. In the expressions for the reduction to the standard system (Crawford \& Mander 1966) we introduced two terms, one for the hour angle and another for the declination, to correct for the flexure error. The corrected data were used as input to compute the final instrumental catalog, shown in Table 3 , again by minimizing the merit function. It is known that the $\beta$ transformation to the standard is different for $\mathrm{B}-\mathrm{A}$ stars and for $\mathrm{A}-\mathrm{G}$; the transformation to the standard values (Perry et al. 1987) of our stars were made forcing all the stars with parameters computed for A-G stars because our sample includes only late-type stars. In Tables 4 and 5 we show standard $\beta$ values for standard and program stars respectively.

on atmospheric extinction have been well studied in relation with photometric observations (Rufener 1986; Grothues \& Gochermann 1992; Manfroid 1992). Results of these studies pointed out that a color dependent increase of the extinction coefficients as well as a great variability of the coefficients can be measured after eruptions, with effects slowly decreasing in the months following the explosion. These effects appear after a time strongly dependent on the geographical coordinates of the site of eruption and of the site of observation, as well as on metheorological factors. Our efforts to search for effects ofthe Pinatubo eruption in our data produced no results, justifying our choice of a single extinction coefficient per night.
Typical standard deviations for stars with $V \leq 10$ are $0.01,0.01,0.01,0.015$ and 0.02 for $V,(b-y), m_{1}, c_{1}$ and $\beta$ respectively; for fainter stars a value of 0.03 is a realistic evaluation of the uncertainty of the data for all colors. Some very large values of the standard deviation, specially in $V$, was found for some binary systems, indicating possible variability.

\section{Results}

Figures 1a and $1 \mathrm{~b}$ show plots of $c_{1}$ and $m_{1}$ vs. $b-y$ for our program stars. Continuous lines indicate the standard main sequence for F (Crawford 1975) and G-M (Olsen 1984) stars. The dashed line connects data obtained by Perry et al. (1987) for 13 class III stars; this line shows the region populated by metal rich giants (Ardeberg \& Lindgren 1981). The stars we have used to define the class III region are reported in Table 6 together with their $[\mathrm{Fe} / \mathrm{H}]$ abundance (Cayrel de Strobel et al. 1992). We have assumed that the effects of interstellar reddening are negligible because, given the typical sensitivity of Einstein $\mathrm{X}$-ray observations and the activity level of a very young star, all the EMSS coronal sources should lie at a distance of $100 \mathrm{pc}$ or less, as it is in fact confirmed by the photometric parallax of the stars in our sample, as well as by the good agreement we find between their $b-y$ and $\beta$ indices. As discussed earlier, the luminosity class of the sample stars was determined during the transformation to the standard values, using the $(b-y)$ and $c_{1}$ indices. The spectral types classification was performed using the $\beta$ and $(b-y)$ indices for $\mathrm{F}$ dwarfs, following Crawford (1975, Table VII), and the $(b-y)$ index alone for $\mathrm{G}-\mathrm{K}$ dwarf, following Olsen (1984, Tables V and VI). For class III stars, the $(b-y)$ index was used irrespective of spectral type, following Ardeberg \& Lindgren (1985, Fig. 2). The diagram shown in panel (a) of Fig. 1 allows us to clearly separate dwarfs from giants. Note that we have classified as class III some stars having a very high lithium abundance, close to the typical "primordial" value for Pop. I objects, which are unlikely to be evolved objects, and are much more likely to be pre-main sequence objects.

The $m_{1}$ vs. $b-y$ diagram of Fig. $1 \mathrm{~b}$ is normally used to estimate the stellar metallicity by measuring the quantity $\delta m_{1}$, defined as $m_{1 \text { (Hyades) }}-m_{1 \text { (obs) }}$. Inspection of Fig. $1 \mathrm{~b}$ shows that most of the cooler stars (both dwarfs and giants) in our sample have $m_{1}$ values which are typical of metal poor stars. Some of the main sequence stars in the sample have a $\delta m_{1}$ value large enough to position them in the region normally occupied by giants.

From Fig. 1b it is evident that the deviation from the standard solar-metallicity sequence for the stars in our sample has a definite color dependence. This is better shown in Fig. 2, where the quantity $\delta m_{1(b-y)}$, defined according to Crawford (1975) as $m_{1, \text { standard }}-m_{1, \text { star is plot- }}$ ted versus the color index $b-y$. Different symbols identify stars of different luminosity class, as determined from the 
Table 3. Instrumental catalog for the SAT in August 1991. The number of observations used in the minimization phases for each color is $n$; $\operatorname{Res}_{\text {index }}=\sum_{i}\left(\operatorname{res}_{i}\right)^{2} /(i-1)$ where $i$ is the running index of the observations

\begin{tabular}{|c|c|c|c|c|c|c|c|c|c|c|c|c|c|c|c|}
\hline Name & $y$ & $n$ & $b-y$ & $n$ & $m_{1}$ & $n$ & $c_{1}$ & $n$ & $\beta$ & $n$ & $\operatorname{Res}_{V}$ & $\operatorname{Res}_{b-y}$ & $\operatorname{Res}_{m_{1}}$ & $\operatorname{Res}_{c_{1}}$ & $\operatorname{Res}_{\beta}$ \\
\hline HR100 & 3.959 & 18 & 0.091 & 18 & 0.202 & 18 & 0.944 & 16 & 2.815 & 5 & 0.009 & 0.005 & 0.004 & 0.006 & 0.006 \\
\hline HR531 & 4.651 & 6 & 0.212 & 9 & 0.184 & 9 & 0.661 & 7 & 2.737 & 6 & 0.007 & 0.005 & 0.005 & 0.009 & 0.012 \\
\hline HR672 & 5.604 & 6 & 0.371 & 6 & 0.178 & 6 & 0.417 & 6 & 2.646 & 1 & 0.008 & 0.006 & 0.007 & 0.008 & - \\
\hline HR962 & 5.066 & 6 & 0.362 & 9 & 0.173 & 9 & 0.430 & 8 & - & - & 0.005 & 0.004 & 0.006 & 0.007 & - \\
\hline HR1024 & 6.208 & 6 & 0.449 & 12 & 0.189 & 12 & 0.312 & 7 & - & - & 0.004 & 0.004 & 0.004 & 0.008 & - \\
\hline HR1089 & 6.484 & 9 & 0.412 & 12 & 0.173 & 12 & 0.452 & 10 & 2.648 & 7 & 0.005 & 0.004 & 0.005 & 0.009 & 0.010 \\
\hline HR1552 & 3.690 & 3 & -0.043 & 3 & 0.062 & 3 & 0.198 & 3 & - & - & 0.013 & 0.004 & 0.006 & 0.005 & - \\
\hline HR2106 & - & - & - & - & - & - & - & - & 2.644 & 3 & - & - & - & - & 0.010 \\
\hline HR4695 & 4.959 & 3 & 0.713 & 3 & 0.504 & 3 & 0.354 & 3 & - & - & 0.006 & 0.004 & 0.006 & 0.008 & - \\
\hline HR5168 & 4.237 & 15 & 0.246 & 15 & 0.168 & 15 & 0.554 & 15 & 2.705 & 6 & 0.006 & 0.005 & 0.006 & 0.007 & 0.005 \\
\hline HR5511 & 3.742 & 12 & 0.006 & 12 & 0.144 & 12 & 1.112 & 11 & 2.818 & 6 & 0.009 & 0.005 & 0.007 & 0.008 & 0.007 \\
\hline HR5530 & 5.158 & 12 & 0.270 & 12 & 0.150 & 12 & 0.518 & 12 & 2.697 & 6 & 0.005 & 0.005 & 0.008 & 0.009 & 0.007 \\
\hline HR5660 & 4.920 & 15 & 0.249 & 16 & 0.133 & 16 & 1.374 & 16 & 2.738 & 4 & 0.006 & 0.004 & 0.006 & 0.009 & 0.005 \\
\hline HR5868 & 4.419 & 13 & 0.384 & 13 & 0.184 & 13 & 0.374 & 13 & 2.636 & 4 & 0.008 & 0.004 & 0.006 & 0.008 & 0.008 \\
\hline HR5997 & 4.317 & 16 & 0.514 & 16 & 0.301 & 16 & 0.381 & 14 & 2.615 & 4 & 0.007 & 0.004 & 0.006 & 0.006 & 0.011 \\
\hline HR6332 & - & - & - & - & - & - & - & - & 2.855 & 6 & - & - & - & - & 0.011 \\
\hline HR6458 & - & - & - & - & - & - & - & - & 2.624 & 6 & - & - & - & - & 0.010 \\
\hline HR6723 & 4.443 & 18 & 0.036 & 18 & 0.133 & 18 & 1.128 & 18 & 2.831 & 6 & 0.008 & 0.005 & 0.006 & 0.005 & 0.009 \\
\hline HR7254 & 4.117 & 13 & 0.024 & 13 & 0.181 & 13 & 1.091 & 14 & 2.854 & 6 & 0.008 & 0.004 & 0.006 & 0.007 & 0.011 \\
\hline HR7858 & 5.391 & 13 & 0.027 & 13 & 0.201 & 13 & 1.023 & 12 & 2.877 & 4 & 0.007 & 0.006 & 0.008 & 0.008 & 0.004 \\
\hline HR8060 & 4.859 & 19 & 0.089 & 19 & 0.190 & 19 & 0.983 & 19 & 2.826 & 6 & 0.005 & 0.004 & 0.006 & 0.007 & 0.010 \\
\hline HR8313 & 4.305 & 10 & 0.699 & 10 & 0.513 & 10 & 0.202 & 10 & - & - & 0.007 & 0.004 & 0.004 & 0.004 & - \\
\hline HR8431 & 4.504 & 1 & 0.031 & 1 & 0.169 & 1 & 1.105 & 1 & - & - & - & - & - & - & - \\
\hline HR 8729 & 5.446 & 4 & 0.415 & 4 & 0.215 & 4 & 0.374 & 4 & - & - & 0.009 & 0.006 & 0.006 & 0.008 & - \\
\hline HR8848 & 4.012 & 19 & 0.264 & 19 & 0.154 & 19 & 0.560 & 19 & 2.669 & 5 & 0.009 & 0.003 & 0.006 & 0.008 & 0.010 \\
\hline HR8969 & 4.129 & 9 & 0.333 & 9 & 0.156 & 9 & 0.413 & 9 & 2.644 & 6 & 0.008 & 0.005 & 0.008 & 0.008 & 0.011 \\
\hline
\end{tabular}

Table 4. Mean values and standard deviations for standard stars in the Crawford-Barnes (uvby) and Crawford-Mander ( $\beta$ ) systems. An asterisk in column 12 (Notes) indicates stars which are not $\beta$ standards. The few B stars present in Table 3 only with $\beta$ values are not reported here because an $\mathrm{A}-\mathrm{F}$ transformation to standard was applied to all stars

\begin{tabular}{|c|c|c|c|c|c|c|c|c|c|c|c|}
\hline Name & $\bar{V}$ & $b-y$ & $m_{1}$ & $c_{1}$ & $\bar{\beta}$ & $\sigma_{V}$ & $\sigma_{b-y}$ & $\sigma_{m_{1}}$ & $\sigma_{c_{1}}$ & $\sigma_{\beta}$ & Notes \\
\hline HR100 & 3.942 & 0.088 & 0.205 & 0.912 & 2.846 & 0.011 & 0.005 & 0.006 & 0.005 & 0.008 & \\
\hline HR531 & 4.650 & 0.209 & 0.188 & 0.641 & 2.741 & 0.008 & 0.006 & 0.008 & 0.010 & 0.015 & \\
\hline HR672 & 5.609 & 0.372 & 0.179 & 0.413 & 2.623 & 0.008 & 0.007 & 0.011 & 0.005 & - & \\
\hline HR962 & 5.075 & 0.363 & 0.180 & 0.421 & 2.627 & 0.013 & 0.003 & 0.003 & 0.007 & 0.026 & $*$ \\
\hline HR1024 & 6.212 & 0.448 & 0.203 & 0.301 & 2.571 & 0.004 & 0.004 & 0.008 & 0.009 & 0.018 & * \\
\hline HR1089 & 6.493 & 0.410 & 0.188 & 0.444 & 2.627 & 0.005 & 0.003 & 0.005 & 0.009 & 0.013 & \\
\hline HR1552 & 3.688 & -0.052 & 0.069 & 0.141 & - & 0.013 & 0.004 & 0.006 & 0.005 & - & \\
\hline HR4695 & 4.971 & 0.721 & 0.472 & 0.502 & 2.560 & 0.003 & 0.001 & 0.006 & 0.007 & 0.015 & $*$ \\
\hline HR5168 & 4.221 & 0.246 & 0.172 & 0.534 & 2.702 & 0.007 & 0.005 & 0.006 & 0.009 & 0.006 & \\
\hline HR5511 & 3.737 & -0.004 & 0.150 & 1.074 & 2.841 & 0.011 & 0.004 & 0.007 & 0.008 & 0.009 & \\
\hline HR5530 & 5.149 & 0.266 & 0.157 & 0.500 & 2.689 & 0.005 & 0.005 & 0.009 & 0.009 & 0.009 & \\
\hline HR5660 & 4.925 & 0.246 & 0.141 & 1.367 & 2.744 & 0.025 & 0.005 & 0.006 & 0.010 & 0.006 & \\
\hline HR5868 & 4.427 & 0.380 & 0.192 & 0.366 & 2.607 & 0.008 & 0.005 & 0.007 & 0.008 & 0.010 & \\
\hline HR5997 & 4.314 & 0.522 & 0.282 & 0.453 & 2.584 & 0.007 & 0.004 & 0.006 & 0.005 & 0.014 & \\
\hline HR6723 & 4.436 & 0.026 & 0.140 & 1.093 & 2.857 & 0.013 & 0.005 & 0.007 & 0.006 & 0.013 & \\
\hline HR7254 & 4.096 & 0.020 & 0.185 & 1.056 & 2.896 & 0.009 & 0.003 & 0.006 & 0.007 & 0.014 & \\
\hline HR7858 & 5.389 & 0.019 & 0.203 & 0.989 & 2.917 & 0.010 & 0.006 & 0.008 & 0.008 & 0.005 & \\
\hline HR8060 & 4.857 & 0.084 & 0.193 & 0.953 & 2.856 & 0.012 & 0.004 & 0.005 & 0.007 & 0.013 & \\
\hline HR8313 & 4.328 & 0.703 & 0.483 & 0.358 & 2.606 & 0.007 & 0.003 & 0.003 & 0.008 & 0.023 & $*$ \\
\hline HR8431 & 4.489 & 0.028 & 0.172 & 1.070 & - & - & - & - & - & - & \\
\hline HR8729 & 5.456 & 0.411 & 0.239 & 0.363 & 2.606 & 0.004 & 0.005 & 0.004 & 0.010 & 0.029 & $*$ \\
\hline HR 8848 & 3.991 & 0.267 & 0.158 & 0.543 & 2.661 & 0.012 & 0.004 & 0.006 & 0.008 & 0.012 & \\
\hline HR8969 & 4.135 & 0.328 & 0.165 & 0.400 & 2.620 & 0.008 & 0.004 & 0.004 & 0.009 & 0.014 & \\
\hline
\end{tabular}

analysis of the $c_{1}$ index. The dashed line indicates the expected $\delta m_{1}$ value of giants with respect to the main sequence. Note that some stars have a $\delta m_{1}$ comparable with that observed in halo stars (Olsen 1984; Schuster \& Nissen 1989); given that most other characteristics of the EMSS coronal source population point toward it being mostly composed of young (i.e. close to ZAMS) sources (Favata et al. 1993, 1995), the large metal deficiency implied by the $m_{1}$ index taken at face value seems very unlikely.

\section{Discussion}

Given the magnitude of the observed $\delta m_{1}$ in our sample of stars and their belonging to a young, active population, the observed behavior cannot be entirely explained in terms of a real metal deficiency. The dependence of the $m_{1}$ deficiency on the effective temperature seems to indicate a real peculiarity of the atmospheres of our active stars with respect to "normal" stars, similar to that observed in active binaries by Giménez et al. (1991). These 
Table 5. Spectral type, derived by photometry discussed in the text, and mean values and standard deviations for program stars in the Crawford \& Barnes (uvby) and Crawford \& Mander $(\beta)$ systems. The number of observations for the $V$ magnitude and colors are $n_{y}$ and $n_{u v b}$, respectively. A 9.999 in the $\sigma$ columns indicates stars with only one observation

\begin{tabular}{|c|c|c|c|c|c|c|c|c|c|c|c|c|c|c|}
\hline N. & Sp. Type & $V$ & $n_{y}$ & $b-y$ & $m_{1}$ & $c_{1}$ & $n_{u v b}$ & $\beta$ & $n_{\beta}$ & $\sigma_{V}$ & $\sigma_{b-y}$ & $\sigma_{m_{1}}$ & $\sigma_{c_{1}}$ & $\sigma_{\beta}$ \\
\hline 1 & F5V & 8.620 & 6 & 0.291 & 0.148 & 0.460 & 6 & 2.663 & 6 & 0.008 & 0.008 & 0.009 & 0.011 & 0.022 \\
\hline 2 & G0V & 7.500 & 15 & 0.371 & 0.182 & 0.312 & 15 & 2.607 & 6 & 0.106 & 0.007 & 0.009 & 0.010 & 0.021 \\
\hline 3 & $\mathrm{~K} 0 \mathrm{~V}$ & 8.454 & 9 & 0.479 & 0.251 & 0.268 & 9 & 2.575 & 6 & 0.030 & 0.008 & 0.009 & 0.011 & 0.009 \\
\hline 4 & F5V & 6.910 & 12 & 0.304 & 0.151 & 0.393 & 12 & 2.649 & 6 & 0.014 & 0.005 & 0.006 & 0.009 & 0.018 \\
\hline 5 & K3V & 5.619 & 6 & 0.504 & 0.397 & 0.256 & 6 & 2.527 & 6 & 0.163 & 0.005 & 0.009 & 0.015 & 0.023 \\
\hline 6 & G8V & 8.916 & 6 & 0.453 & 0.248 & 0.275 & 9 & 2.577 & 6 & 0.010 & 0.005 & 0.007 & 0.013 & 0.024 \\
\hline 7 & $\mathrm{~K} 2 \mathrm{~V}$ & 8.100 & 3 & 0.535 & 0.484 & 0.263 & 3 & 2.563 & 3 & 0.003 & 0.001 & 0.005 & 0.008 & 0.010 \\
\hline 8 & G0III & 10.310 & 3 & 0.473 & 0.275 & 0.403 & 3 & - & - & 0.019 & 0.006 & 0.010 & 0.017 & - \\
\hline 9 & F8V & 8.916 & 3 & 0.350 & 0.156 & 0.377 & 3 & 2.611 & 3 & 0.006 & 0.001 & 0.006 & 0.010 & 0.020 \\
\hline 10 & $\mathrm{~K} 1 \mathrm{~V}$ & 9.626 & 3 & 0.492 & 0.270 & 0.277 & 3 & - & - & 0.001 & 0.006 & 0.011 & 0.007 & 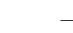 \\
\hline 11 & KOV & 8.442 & 3 & 0.489 & 0.329 & 0.320 & 6 & 2.581 & 6 & 0.006 & 0.008 & 0.009 & 0.009 & 0.013 \\
\hline 12 & K5V & 10.312 & 3 & 0.635 & 0.549 & 0.197 & 3 & - & - & 0.007 & 0.006 & 0.006 & 0.021 & - \\
\hline 13 & G5V & 10.504 & 3 & 0.417 & 0.216 & 0.298 & 6 & 2.583 & 3 & 0.007 & 0.009 & 0.017 & 0.037 & 0.022 \\
\hline 14 & F7V & 10.133 & 3 & 0.337 & 0.164 & 0.421 & 6 & 2.638 & 6 & 0.006 & 0.007 & 0.011 & 0.016 & 0.031 \\
\hline 15 & $\mathrm{~K} 4 \mathrm{~V}$ & 9.143 & 3 & 0.627 & 0.554 & 0.152 & 6 & 2.508 & 6 & 0.001 & 0.007 & 0.008 & 0.019 & 0.023 \\
\hline 16 & F7V & 10.314 & 3 & 0.356 & 0.136 & 0.431 & 6 & 2.650 & 3 & 0.002 & 0.011 & 0.021 & 0.017 & 0.053 \\
\hline 17 & KOIII & 7.287 & 6 & 0.684 & 0.440 & 0.384 & 6 & 2.562 & 6 & 0.001 & 0.004 & 0.004 & 0.009 & 0.032 \\
\hline 18 & K2IV & 10.656 & 6 & 0.516 & 0.268 & 0.318 & 6 & 2.575 & 3 & 0.043 & 0.013 & 0.020 & 0.022 & 0.025 \\
\hline 19 & F5V & 6.346 & 6 & 0.276 & 0.163 & 0.492 & 6 & 2.693 & 3 & 0.005 & 0.004 & 0.005 & 0.007 & 0.020 \\
\hline 20 & F6V & 12.059 & 3 & 0.320 & 0.131 & 0.430 & 3 & - & - & 0.012 & 0.004 & 0.014 & 0.017 & - \\
\hline 21 & G8III & 10.645 & 3 & 0.584 & 0.141 & 0.428 & 6 & - & - & 0.003 & 0.005 & 0.010 & 0.021 & - \\
\hline 22 & K1V & 10.485 & 3 & 0.491 & 0.281 & 0.258 & 3 & - & - & 0.004 & 0.003 & 0.010 & 0.020 & - \\
\hline 23 & K2III & 7.469 & 3 & 0.805 & 0.523 & 0.322 & 3 & - & - & 0.001 & 0.002 & 0.002 & 0.008 & - \\
\hline 24 & KOIII & 10.770 & 3 & 0.640 & 0.260 & 0.488 & 3 & - & - & 0.007 & 0.049 & 0.019 & 0.068 & - \\
\hline 25 & G8V & 9.732 & 30 & 0.456 & 0.231 & 0.266 & 30 & 2.568 & 6 & 0.184 & 0.010 & 0.011 & 0.018 & 0.021 \\
\hline 26 & F5V & 6.913 & 12 & 0.289 & 0.169 & 0.472 & 12 & 2.660 & 6 & 0.006 & 0.006 & 0.007 & 0.009 & 0.029 \\
\hline 27 & $\mathrm{G} 2 \mathrm{~V}$ & 7.358 & 15 & 0.392 & 0.206 & 0.329 & 15 & 2.584 & 6 & 0.015 & 0.005 & 0.009 & 0.010 & 0.032 \\
\hline 28 & $\mathrm{~F} 4 \mathrm{~V}$ & 5.818 & 15 & 0.268 & 0.169 & 0.540 & 15 & 2.682 & 6 & 0.009 & 0.005 & 0.007 & 0.009 & 0.040 \\
\hline 29 & G3V-IV & 10.953 & 9 & 0.409 & 0.175 & 0.365 & 9 & 2.619 & 3 & 0.013 & 0.007 & 0.009 & 0.035 & 0.023 \\
\hline 30 & $\mathrm{~K} 4 \mathrm{~V}$ & 9.737 & 12 & 0.605 & 0.554 & 0.194 & 30 & 2.548 & 3 & 0.009 & 0.008 & 0.009 & 0.015 & 0.032 \\
\hline 31 & GoV & 11.185 & 3 & 0.371 & 0.134 & 0.398 & 3 & - & - & 0.017 & 0.015 & 0.014 & 0.011 & - \\
\hline 32 & KOIII & 7.333 & 13 & 0.651 & 0.335 & 0.422 & 13 & 2.549 & 6 & 0.029 & 0.005 & 0.005 & 0.012 & 0.019 \\
\hline 33 & $\mathrm{~F} 2 \mathrm{~V}$ & 6.584 & 10 & 0.239 & 0.158 & 0.506 & 10 & 2.696 & 3 & 0.022 & 0.006 & 0.006 & 0.010 & 0.023 \\
\hline 34 & K1III & 8.101 & 1 & 0.745 & 0.491 & 0.398 & 1 & - & - & 9.999 & 9.999 & 9.999 & 9.999 & - \\
\hline 35 & G6V-IV & 8.935 & 16 & 0.438 & 0.210 & 0.311 & 16 & 2.588 & 6 & 0.014 & 0.006 & 0.009 & 0.015 & 0.024 \\
\hline 36 & KOIII & 11.424 & 7 & 0.704 & 0.356 & 0.348 & 7 & - & - & 0.022 & 0.013 & 0.019 & 0.043 & - \\
\hline 37 & K1III & 11.278 & 4 & 0.728 & 0.414 & 0.324 & 4 & - & - & 0.006 & 0.007 & 0.018 & 0.020 & - \\
\hline 38 & K5V & 9.154 & 12 & 0.666 & 0.722 & 0.115 & 12 & 2.521 & 6 & 0.009 & 0.005 & 0.010 & 0.020 & 0.018 \\
\hline 39 & K0III & 10.535 & 6 & 0.657 & 0.303 & 0.376 & 6 & 2.578 & 6 & 0.019 & 0.012 & 0.015 & 0.033 & 0.034 \\
\hline 40 & K5V & 11.803 & 7 & 0.628 & 0.385 & 0.178 & 7 & - & - & 0.022 & 0.013 & 0.030 & 0.049 & - \\
\hline 41 & G2V & 9.094 & 15 & 0.396 & 0.163 & 0.348 & 15 & 2.619 & 6 & 0.018 & 0.004 & 0.009 & 0.014 & 0.006 \\
\hline 42 & G2V-IV & 11.579 & 9 & 0.386 & 0.164 & 0.396 & 9 & - & - & 0.052 & 0.022 & 0.023 & 0.019 & 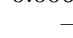 \\
\hline 43 & F6V & 11.444 & 13 & 0.329 & 0.146 & 0.396 & 13 & - & - & 0.015 & 0.016 & 0.023 & 0.026 & - \\
\hline 44 & K3V & 11.418 & 1 & 0.581 & 0.415 & 0.172 & 1 & - & - & 9.999 & 9.999 & 9.999 & 9.999 & - \\
\hline 45 & G8III & 10.263 & 19 & 0.576 & 0.203 & 0.439 & 19 & 2.616 & 6 & 0.035 & 0.013 & 0.014 & 0.029 & 0.035 \\
\hline 46 & G8V & 9.842 & 19 & 0.454 & 0.222 & 0.286 & 19 & 2.563 & 6 & 0.018 & 0.010 & 0.012 & 0.009 & 0.017 \\
\hline 47 & $\mathrm{~K} 2 \mathrm{~V}$ & 11.366 & 4 & 0.514 & 0.321 & 0.214 & 4 & - & - & 0.081 & 0.006 & 0.014 & 0.038 & - \\
\hline 48 & $\mathrm{~F} 4 \mathrm{~V}$ & 7.295 & 10 & 0.295 & 0.161 & 0.487 & 10 & 2.686 & 6 & 0.008 & 0.004 & 0.008 & 0.009 & 0.012 \\
\hline 49 & G4V & 10.679 & 6 & 0.432 & 0.205 & 0.296 & 6 & 2.608 & 3 & 0.019 & 0.007 & 0.004 & 0.027 & 0.020 \\
\hline
\end{tabular}

Table 6. Standard uvby values (Perry et al. 1987) and $[\mathrm{Fe} / \mathrm{H}]$ (Cayrel de Strobel et al. 1992) abundances for the class III sequence

\begin{tabular}{|llccccc|}
\hline Name & Sp. Type & $V$ & $b-y$ & $m_{1}$ & $c_{1}$ & {$[\mathrm{Fe} / \mathrm{H}]$} \\
\hline HR8665 & F6III-IV & 4.190 & 0.330 & 0.147 & 0.407 & $-0.05 /-0.28$ \\
HR2662 & G0III-IV & 6.294 & 0.359 & 0.192 & 0.402 & \\
HR3815 & G8III & 5.410 & 0.473 & 0.304 & 0.372 & 0.00 \\
HR5997 & G3II-III & 4.316 & 0.522 & 0.285 & 0.448 & \\
HR1030 & G6III & 3.613 & 0.547 & 0.333 & 0.426 & \\
HR2985 & G8III & 3.570 & 0.573 & 0.379 & 0.398 & \\
HR1373 & K0III & 3.759 & 0.597 & 0.424 & 0.405 & \\
HR4392 & G7.5III & 4.989 & 0.610 & 0.416 & 0.396 & +0.06 \\
HR617 & K2IIIab & 2.000 & 0.696 & 0.526 & 0.395 & $-0.21 /-0.08 /-0.25 /-0.29$ \\
HR6603 & K2III & 2.760 & 0.719 & 0.553 & 0.451 & +0.14 \\
HR5947 & K2IIIab & 4.150 & 0.751 & 0.570 & 0.414 & \\
HR3003 & K5III & 4.848 & 0.895 & 0.735 & 0.451 & \\
HR1457 & K5III & 0.860 & 0.955 & 0.814 & 0.373 & $0.00 /-0.10 /-0.33 /-0.14$ \\
\hline
\end{tabular}



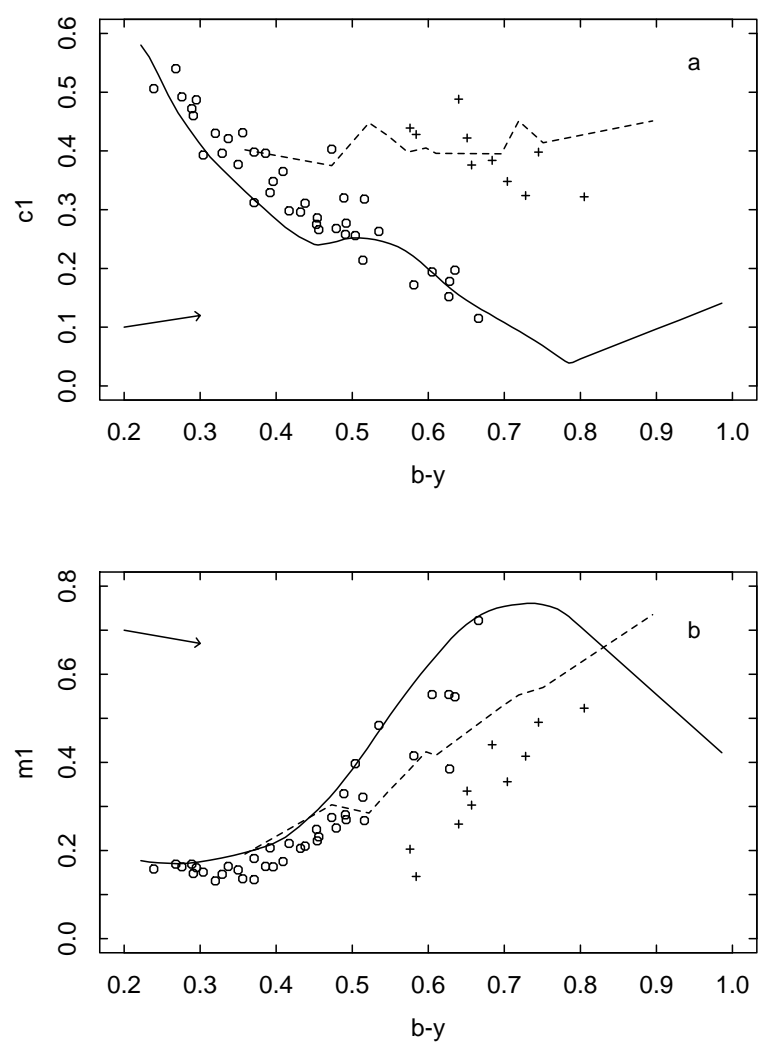

Fig. 1. Two-color plots of program stars: a) $c_{1}$ versus $(b-y)$, b) $m_{1}$ versus $(b-y)$. Panel a) shows a clear separation among luminosity classes III and V. Pluses indicate stars we classify as giants, while empty symbols indicate main sequence stars. Solid lines indicate the standard main sequence for $F$ (Crawford 1975) and G-M (Olsen 1984) stars. Dashed lines connect data obtained by Perry et al. (1987) for 13 class III stars. Arrows indicate the reddening vector

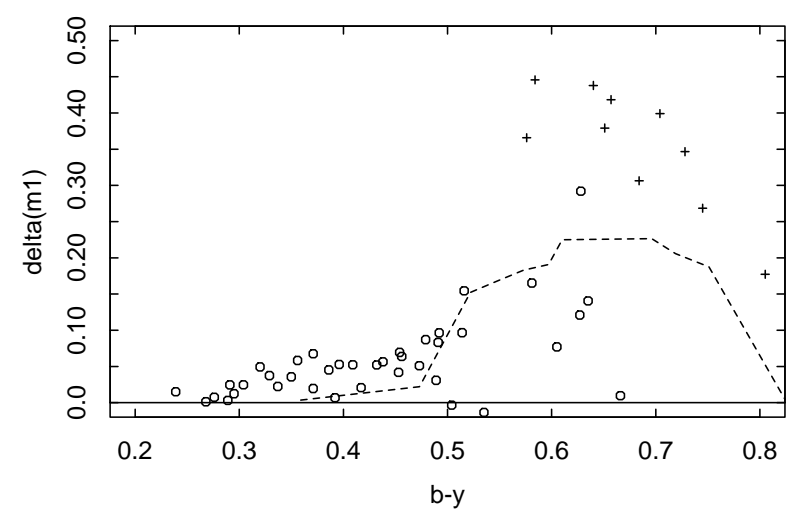

Fig. 2. $\delta m_{1(b-y)}$ versus color for program stars; symbols and lines as in Fig. 1. The dashed line indicates the expected value of $\delta m_{1}$ for normal giants authors find that the observed $m_{1}$ anomaly becomes larger in cooler binary systems and that, when individual components are resolved, the metal deficiency implied by the observed $\delta m_{1}$ in the cooler, active component is consistently larger than the deficiency implied in the hotter, non-active component. This is incompatible with an effect actually due to anomalous chemical abundances, as the abundance of both binary components, which share a common evolutionary history, is expected to be the same. Giménez et al. (1991) conclude that the observed $m_{1}$ index is in fact affected by the chromospheric activity level of the stars, as suggested by their observed relation between the amplitude of magnitude variations (assuming that they are due to the change of spot surface coverage and therefore related to the activity level) and the size of $\delta m_{1}$.

Additional evidence of apparent metal deficiencies induced by stellar activity is present in the literature since some time: the influence of stellar activity on the observed Strömgren photometric indices was already suggested by Giampapa et al. (1979), based on their observations of quiet and active zones on the Sun surface. Active solar regions show lower values of the $m_{1}$ index than do quiet solar regions, so that the implied $[\mathrm{Fe} / \mathrm{H}]$ value is $\sim 35 \%$ lower in active than in quiet regions. Also, a long observational campaign on AB Dor (Jetsu et al. 1990), a very young active $\mathrm{K}$ star that shows visual variability probably due to the presence of spots, has shown that $\mathrm{AB}$ Dor has a value of the $m_{1}$ index lower than expected for a young, close to ZAMS, star. Finally, Basri et al. (1989) compared some photospheric absorption lines in active and quiet stars of the same effective temperature, finding evidence of emission in the cores of these lines, which increased with the level of activity. This emission, by decreasing the equivalent width of the absorption lines, could perhaps mimic the appearance of lower metallicity.

Our data confirm that activity affects Strömgren photometric indices also in non binary systems, at least for stars redder than $b-y \approx 0.450$, corresponding approximately to spectral type G8. In Fig. 3 we plot, for the dwarfs in our sample, the observed $\delta m_{1}$ versus the activity indicator $f_{\mathrm{X}} / f_{\mathrm{V}}$. The $f_{\mathrm{X}} / f_{\mathrm{V}}$ index is computed in the $0.3-3.5 \mathrm{keV}$ band, from the Einstein observations of Gioia et al. (1990) and from our optical photometry. Different symbols indicate stars earlier and later than G8. It is evident that higher values of $\delta m_{1}$ are correlated with higher activity levels. Also, the cooler stars show an increase in the apparent metal deficiency with respect to the hotter stars.

The large number of binaries or suspected binaries present in our sample do not behave differently from the single stars in the $\delta m_{1(b-y)}$ versus $\left(f_{\mathrm{X}} / f_{\mathrm{V}}\right)$ plane, as seen in Fig. 3. Also, while our sample is likely to contain a few unrecognized binaries, they are unlikely the cause of the observed peculiar behavior of the $m_{1}$ index: following 


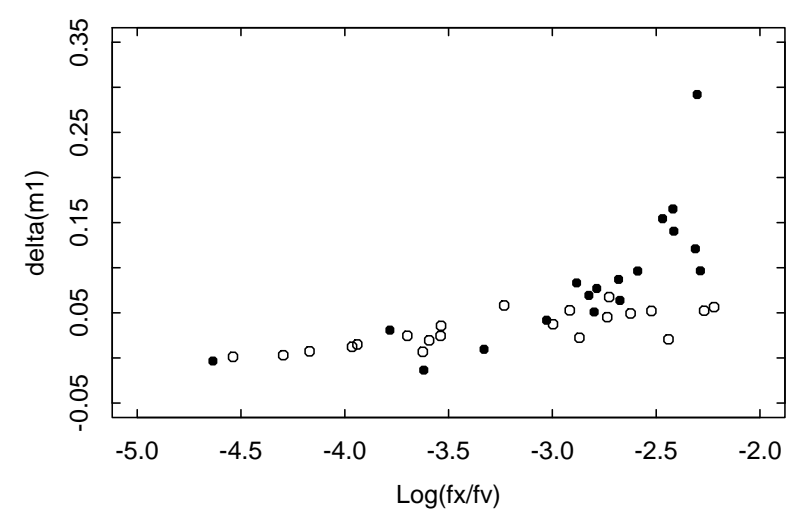

Fig. 3. $\delta m_{1(b-y)}$ versus $\left(f_{\mathrm{X}} / f_{\mathrm{V}}\right)$ for our program stars. Stars earlier and later than G8 are indicated by empty and filled symbols respectively. A clear relation between activity levels and $m_{1}$ index deficiency is evident for the cooler stars

the reasoning of Hilditch et al. (1976), Knude (1978) and Giménez et al. (1991), binary systems in which both the components are dwarfs can change their apparent effective temperature by the equivalent of a few spectral subtypes, with all other indices remaining unchanged, while binary systems with at least one evolved component show a $c_{1}$ index typical of giants. Therefore the possible presence of unrecognized binaries could not affect $m_{1}$ without affecting $c_{1}$.

We have verified that activity does not affect the $c_{1}$ index for the stars in our sample (an effect which would make the luminosity class determination unreliable). To this end we have transformed all instrumental colors to the standard colors assuming that all the stars in the sample were main sequence stars, computing for every star the quantities $\delta c_{1(b-y)}=c_{1 \text { (star) }}-c_{1 \text { (main sequence) }}$ for the same color. In the plot of the resulting $\delta c_{1}(b-y)$ versus $f_{\mathrm{X}} / f_{\mathrm{V}}$ no clear dependence of $\delta c_{1(b-y)}$ on activity was found, showing that activity does not affect a star's position in the $c_{1}$ vs. $b-y$ diagram, which can therefore still be used to determine the luminosity class also for very active stars.

The observed anomaly in the observed $m_{1}$ indices seems to be exclusively determined by the activity. Let us consider the case of a star similar to the Sun, but completely covered by active regions. If we assume that the observed anomaly in $m_{1}$ is due to the filling up of metallic lines in the star's spectrum in the $v$ band we can write

$$
\delta m_{1}=m_{1, \mathrm{Q}}-m_{1, \mathrm{~A}}=2.5 \log \left(\frac{f_{\mathrm{A}}}{f_{\mathrm{Q}}}\right)=2.5 \log \left(1+\frac{f_{\mathrm{E}}}{f_{\mathrm{Q}}}\right)
$$

where $m_{1, \mathrm{~A}}$ is the color index observed in the active star, $m_{1, \mathrm{Q}}$ is the index measured on a quiet star of identical spectral type, and $f_{\mathrm{A}}$ is the flux emitted in the $v$ band by the active star, defined as $f_{\mathrm{A}}=f_{\mathrm{E}}+f_{\mathrm{Q}}$, where $f_{\mathrm{E}}$ is the excess flux due to the stellar activity and $f_{\mathrm{Q}}$ is the flux emitted by the quiet star in the $v$ band. If we consider the Sun, substituting $\delta m_{1}=0.027$, the mean of the Giampapa et al. (1979) results, we obtain $f_{\mathrm{E}} / f_{\mathrm{Q}}=0.025$, i.e. the chromosphere of a Sun-like stars completely covered by active regions emits, in the $v$ band, a flux equal to $2.5 \%$ of the flux emitted by the photosphere of a quiet star. Let us now consider a $\mathrm{K} 0$ star completely covered with active regions similar to those discussed above. In this case we have $f_{\mathrm{Q}} \sim 0.24 \cdot f_{\mathrm{Q}_{\odot}}$ due to the differences in effective temperature and stellar radius, while $f_{\mathrm{E}} \sim 0.72 \cdot f_{\mathrm{E}_{\odot}}$ due to the difference in stellar radius only, and assuming that the surface activity flux is independent from spectral type. Hence for a K0 star we can write

$$
\delta m_{1, \mathrm{~K} 0}=2.5 \log \left(1+\frac{f_{\mathrm{E}, \mathrm{K} 0}}{f_{\mathrm{Q}, \mathrm{K} 0}}\right)=2.5 \log \left(1+\frac{0.72 f_{\mathrm{E}_{\odot}}}{0.24 f_{\mathrm{Q}_{\odot}}}\right)
$$

With these assumptions we obtain for a K0 star completely covered by active regions a value of $\delta m_{1}=0.077$. A similar computation for a K5V star, corresponding approximately to the cooler stars in our sample, yields a maximum value $\delta m_{1}=0.267$.

The large $\delta m_{1}$ anomaly in the very active, cool stars of our sample (again, implying metallicities comparable to the ones found in halo stars, following the calibration of Olsen 1984), casts some doubts not only on the reliability of the $m_{1}$ index for metallicity determinations in populations of unknown characteristics, but also on the usage of Strömgren photometry for the segregation of samples of stars on a population basis.

As discussed earlier, our parent sample has been studied in the past to characterize the presence of an excess of yellow stars in the EMSS (Favata et al. 1993, 1995). These high resolution spectroscopic observations have shown that at least a part of this excess is due to the presence of a young population, at least as measured by the lithium abundance. Similar results have been obtained by other authors on different samples selected, as ours, from X-ray or EUV selected surveys (Tagliaferri et al. 1994; Jeffries et al. 1993; Hodgkin \& Pye 1994). In Fig. 4 we show, for the subsample in common with Favata et al. (1993; 1995), a plot of $\delta m_{1}$ versus the lithium abundance. The more lithium rich stars are in general the hotter stars (empty symbols in the figure) in which the lithium depletion is slow (Pinsonneault et al. 1990); for these stars no relationship between the $\delta m_{1}$ anomaly and the lithium abundance is visible. Instead, for $\mathrm{K}$ stars a trend of increasing lithium abundance with increasing $\delta m_{1}$ is visible. The three coolest class III stars of Fig. 4 have been classified as probable pre-main sequence stars by Favata et al. (1993) on the basis of their high lithium abundance. We note that in Fig. 4 these stars seem to follow the same trend of the K main sequence stars. For G stars we cannot observe a similar behavior both because the dependence of $\delta m_{1}$ on activity indicators is small and because the decay time of the lithium abundance is presumably large with respect of the decay time of activity. 


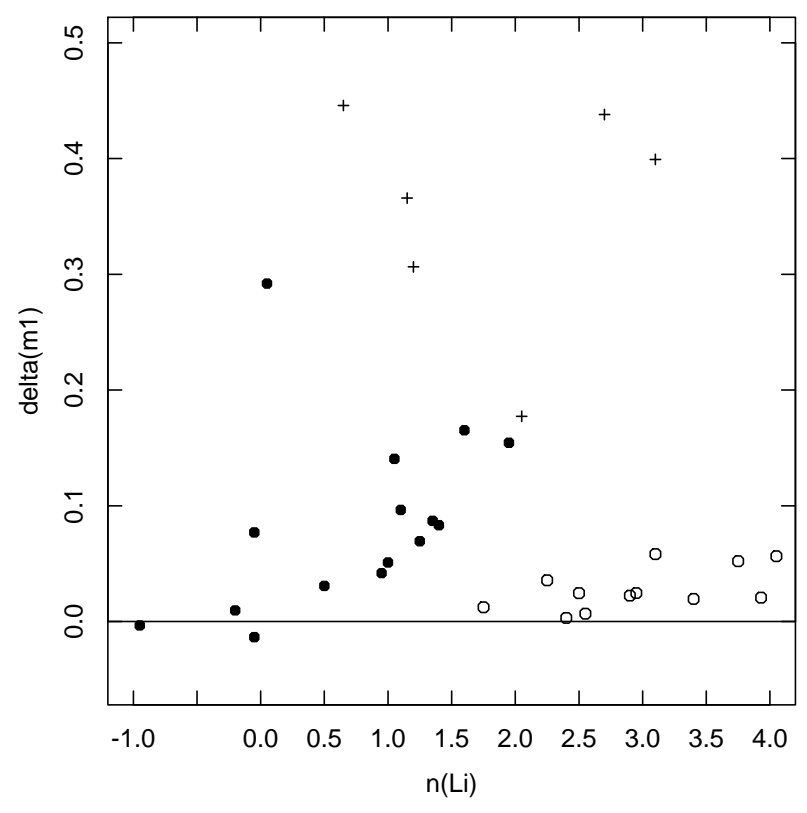

Fig. 4. $\delta m_{1(b-y)}$ versus lithium abundance (in the usual log scale where the hydrogen abundance is set to 12) for program stars. Pluses represent class III stars, empty symbols main sequence stars earlier than G8 and full symbols main sequence stars later than G8. Note as the relationship between $\delta m_{1(b-y)}$ and lithium abundance for the $\mathrm{K}$ stars

In the light of these results and considering that the lithium abundance of $\mathrm{K}$ stars is expected to decay very rapidly with increasing age we can conclude that a high lithium abundance is a reliable sign of young age for $\mathrm{K}$ stars (see also discussion in Favata et al. 1995). Hence, we have a consistent picture in which the activity in $\mathrm{K}$ stars, strictly related with age, affects the $m_{1}$ color index in very young active stars.

\section{Summary and conclusions}

We have reported new photometric observations in the Strömgren-Crawford $u v b y-\beta$ system of a sample of stars detected in X-ray in the Einstein Medium Sensitivity survey. This sample contains an excess of yellow stars with respect to the solar neighborhood stellar population (Favata et al. 1988; Sciortino et al. 1995) attributed to the presence of a young stellar population (Favata et al. 1993, 1995).

Our observations show that stellar positions in the $c_{1}$ vs. $b-y$ diagram are unaffected by activity and that this diagram is still a powerful mean to discriminate between class V and class III stars.

Furthermore main sequence $\mathrm{K}$ stars in our sample show values of $m_{1}$ index lower respect to those of the Hyades main sequence, mimicking an apparent deficiency of metals. We found that the observed behavior cannot be explained by the presence of unrecognized binaries in the sample, and that the size of the observed photometric anomaly increases with stellar activity, as measured by the $f_{\mathrm{X}} / f_{\mathrm{V}}$ ratio, as well as with lithium abundance. Filling-in of the core of metallic lines, due to the high level of activity observed in these stars, is a likely explanation for the $m_{1}$ index behavior, making this index unreliable as metallicity indicator for late type stars. The observed dependence of the $m_{1}$ anomaly on the lithium abundance is interpreted as an effect induced by the age-dependence of activity level for $\mathrm{K}$ stars and the reliable link between high lithium abundance and young age in $\mathrm{K}$ dwarfs.

A similar effect is not observed in G stars where the effect of the activity on $\delta m_{1}$ seems weak. Data are consistent with a picture in which the chromospheric activity, due to active regions similar to the solar active regions covering up to the overall stellar surface, fill up metallic lines mainly in the $v$ band. The different behavior of $\mathrm{G}$ and $\mathrm{K}$ stars is related to the greater ratio $f_{\mathrm{E}} / f_{\mathrm{Q}}$ between the chromospheric (active) and photospheric (quiet) fluxes in the $v$ band for $\mathrm{K}$ stars with respect to $\mathrm{G}$ stars.

Acknowledgements. F.M., G.M. and S.S. acknowledge financial support from ASI (Italian Space Agency), MURST (Ministero della Università e della Ricerca Scientifica e Tecnologica), and GNA-CNR. We would like to thank M. Barbera for his contribution in performing the observations. During this work we have extensively used the Simbad database.

\section{References}

Ardeberg A., Lindgren H., 1981, Rev. Mexicana. Astron. Astrof. 6, 173

Ardeberg A., Lindgren H. 1985, in Calibration of Fundamental Stellar Quantities. In: Hayes D.S., Pasinetti L. and Philip A.G.D. (eds.), IAU Symp. 111, 507

Basri G., Wilcots E., Stout N., 1989, PASP 101, 528

Cayrel de Strobel G., Hauck B., François P., Friel E., Mermillod M., Borde S., 1992, A\&AS 95, 273

Crawford D.L., Mander J., 1966, AJ 71, 114

Crawford D.L., Barnes J.V., 1970, AJ 75, 978

Crawford D.L., 1975, AJ 80, 955

Crawford D.L., 1978, AJ 83, 48

Crawford D.L., 1979, AJ 84, 1858

Favata F., Rosner R., Sciortino S., Vaiana G.S., 1988, ApJ 324, 1010

Favata F., Barbera M., Micela G., Sciortino S., 1993, A\&A 277,428

Favata F., Barbera M., Micela G., Sciortino S., 1995, A\&A 295,147

Favata F., Micela G., Sciortino S., 1995, A\&A 297, L1

Fleming T.A., Gioia I., Maccacaro T., 1989, AJ 98, 692

Giampapa M.S., Worden S.P., Gilliam L.B., 1979, ApJ 229, 1143

Giménez A., Reglero V., de Castro E., Fernández-Figueroa M.J., 1991, A\&A 248, 563

Gioia I., Maccacaro T., Schild R.E., Wolter A., Stocke J.T., 1990, ApJS 72, 567

Gronbech B., Olsen E.H., Strömgren B., 1976, A\&AS 26, 155

Grothues H.G., Gochermann J., 1992, The Messenger 68, 43 
Hilditch R.W., Hill G., Barnes J.V., 1976, MNRAS 176, 175

Hodgkin S.T., Pye J.P., 1994, MNRAS 267, 840

Jeffries R.D., Bromage G.E., 1993, MNRAS 260, 132

Jetsu L., Vilhu O., la Dous C., 1990, A\&AS 85, 1127

Knude J., 1978, A\&AS 33, 347

Manfroid J., Heck A., 1983, A\&A 120, 302

Manfroid J., 1992, A\&A 271, 714

McNamara D.H., Powell J.M., 1985, PASP 97, 1101

Nissen P.E., 1981, A\&A 97, 145

Nissen P.E., Schuster W.J., 1991, A\&A 251, 457

Olsen E.H., 1983, A\&AS 54, 55

Olsen E.H., 1984, A\&AS 57, 443

Olsen E.H., 1988, A\&A 189, 173

Olsen E.H., 1993, A\&AS 102, 89

Olsen E.H., 1994, A\&AS 104, 429

Perry C.L., Olsen E.H., Crawford D.L., 1987, PASP 99, 1184

Pinsonneault M.H., Kawaler S.D., Demarque P., 1990, ApJS 74,501
Pounds K.A., Allan D.J., Barber C., et al., 1993, MNRAS 260, 77

Reglero V., Giménez A., Estela A., Fabregat J., 1990, Astrophys. Space Sci. 169, 263

Rufener F., 1986, A\&A 165, 275

Saxner M., Hammarbäck G., 1985, A\&A 151, 372

Schuster W.J., Nissen P.E., 1988, A\&A 73, 225

Schuster W.J., Nissen P.E., 1989, A\&A 221, 65

Schwarz H.E., Melnick J., 1989, The ESO Users Manual 1990, 151

Sciortino S., Favata F., Micela G., 1995, A\&A 296, 370

Sterken C., Manfroid J., 1992, "Astronomical Photometry - A Guide". Kluwer Academic Publishers, Dordrecht

Stocke J.T., Morris S.L., Gioia I.M., et al., 1991, ApJS 76, 813

Tagliaferri G., Cutispoto G., Pallavicini R., Randich S., Pasquini L., 1994, A\&A 285, 272 